\title{
An Intelligent Optimization Algorithm for Optimal Route Selection for Wireless Sensor Networks
}

\author{
${ }^{1}$ V. Manikandan, ${ }^{1}$ M. Sivaram, ${ }^{2}$ Amin Salih Mohammed and ${ }^{3}$ V. Porkodi \\ ${ }^{1}$ Department of Computer Networking, \\ ${ }^{2}$ College of Engineering and Computer Science, \\ ${ }^{3}$ Department of Information Technology, Lebanese French University, Erbil, Iraq \\ v.manikandan@1fu.edu.krd
}

\begin{abstract}
In Wireless Sensor Networks (WSN), sensor nodes makes use of battery energy, routing and energy consumption along with certain limitations of sensor nodes are considered as a preliminary challenges and problems associated with WSN. In recent times, in WSNs, identification of successive nodes based on the localization and the energy minimization can be achieved by partitioning the nodes into clusters at the central region. In this manner, this research attempts to maintain energy consumption using optimal cuckoo search model in the network nodes. While comparing this with conventional models, the proposed model for energy estimation attains finest efficiency in relation to improvement in network lifetime. In this investigation, cuckoo optimization algorithm with Markov Chain model for successive identification of transition state is performed in WSN environment for identifying the optimal nodes for multi-hop routing and estimating energy. The anticipated strategy is measured in three specific criteria, nodes successive life time, energy utilization, end to end delay with targeted CMMRR algorithm, the residual energy, distance amongst the nodes and cluster head distance is also observed. The outcomes of simulating the anticipated CMMRR method are done in MATLAB environment. The outcomes of the proposed method is compared with the existing techniques like EAR Model, the proposed method shows better trade off $10.4-29 \%$ over prevailing EAR protocol in terms of packet delivery ratio, nodes life time and so on.
\end{abstract}

Key words: WSN, cuckoo search, Markov Model, energy utilization, routing strategy, EAR protocol

\section{INTRODUCTION}

Wireless Sensor Networks (WSN) comprises of set of sensor nodes which has the ability to process, measure and transmit information to nodes in the network by considering certain factors in network environment (Ghaffari, 2014). Sensor nodes have some limitation with regards to radio range, bandwidth, energy and memory, processing capabilities. In large scale scenario of WSN as the energy needed for transmitting the sensed data to base station is higher, therefore, an energy efficient routing protocol is required for performing this task. In association with some specific limitations of WSNs, specifically in realm of routing protocol's energy consumption, there is an essential requirement for constructing a communication routes amongst base station and sensor nodes (Ghaffari and Nobahary, 2015). It has to be noted that certain protocols has to be aware of energy level, subsequently, they may results in optimizing the network lifetime. In hierarchical routing protocols, nodes are partitioned into individual clusters and a node with higher energy level has to be chosen as cluster head. Some features of routing protocols comprises of fault tolerant, reliability, scalability, information accumulation and so on. One significant conventional protocol known as LEACH can be considered as one instance for such protocol type.

There are two stages involved in clustering the traditional LEACH protocol such as setup stage and steady state stage. In the initial stage, every node is chosen randomly with a range of $0-1$. Then, in accordance to selected value, it generates a decision of whether or not it should works as a cluster head in the next successive round. Subsequently, its selection is given to neighbourhood nodes in the cluster (Mottaghinia and Ghaffari, 2016). Following this, other sensor nodes merges with the nearest elected cluster head. The nearest node is figured as Received Signal Strength Indicator (RSSI). Then, successive cluster head generates a time division multiple access schedule for currently generated clusters

Corresponding Author: V. Manikandan, Department of Computer Networking, Lebanese French University, Erbil, Iraq v.manikandan@1fu.edu.krd 


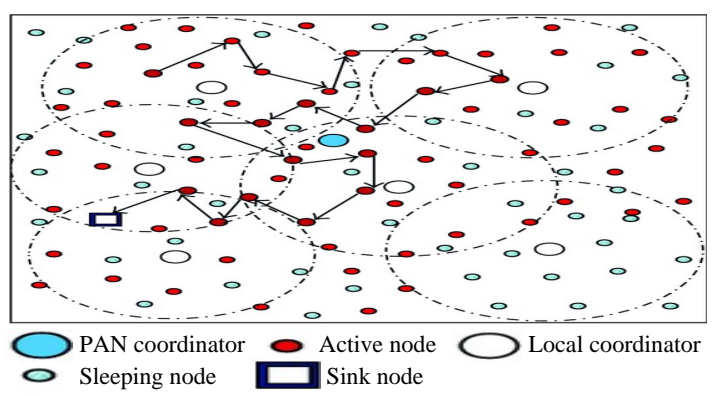

Fig. 1: WSN distributed environment

(Abuarqoub et al., 2017). In next stage, odes of the cluster are formed for producing data to related cluster head with the allocated time slot. Indeed, LEACH is considered as fully probabilistic protocol for selecting cluster head which results in inappropriate election of cluster head (Shokouhifar and Jalali, 2017). Subsequently, this main negative impact of network lifetime with LEACH leads to the design of new routing model. Moreover, as LEACH directly broadcasts data from cluster head to sink, it also shows scalability crisis.

As energy resources in WSNs are restricted, developing an energy efficient routing algorithm in WSNs is measured as a preliminary problem and challenge. It is also identified that clustering is also one amongst the most logical method for improving energy efficiency and for optimizing the WSN lifetime (Mann and Singh, 2017). It has to note that the anticipated model for routing and some traditional methods have some shortcomings which restrict and create problems in real time network scenario. In numerous cases, typical cluster head selection from all sensor nodes in provided network leads to unbalanced cluster production. As well, control factors are generally characterized manually. Moreover, application specification is not generally considered for regulating and adjusting the protocols.

It should be considered that choosing cluster heads is connected with the NP hard optimization problem in wireless sensor networks. The accessible clustering technique is considered and all the clusters are measured as objects that can be modified while enhancing energy efficiency. Moreover, it has to consider that real life problems have hybrid natures and certain complications. Therefore, resolving this crisis intricate with sophisticated algorithm is needed (Fig. 1).

In this research, an effectual routing protocol on cuckoo optimization with a mathematical model known as Markov chain model (CMMRR) protocol is anticipated as energy aware and successive transition state routing. It also considers comprehensive nodes information, i.e., remaining modes energy, distance form base station, intra cluster distance and inter cluster distance for choosing cluster heads. Choosing an optimal cluster head in every round is also performed using fitness function computation. As routing s considered as most important criteria in most WSN applications, three parameters for performance computation and error identification is done using CMMRR which can lead effectual routing and reduced energy consumption amongst sensor nodes. Therefore, the anticipated CMMRR can leads to improved network life time.

Literature review: By Adamu illustrates effectively detects and prevents black hole attacks in WSNs. If there is a chance of black hole attack in the network, our method uses routing through multiple base stations. Otherwise, routing is done through the nearest base station only, in order to reduce energy consumption in WSNs. Also, this research demonstrate that check agents have an important factor in black hole attacks detection in WSNs by decreasing the complexity of the messages and reducing network overhead. Data delivery is ensured due to the use of multiple base stations which gives our method significant improvement compared to the current used methods.

By Kuila and Jana (2014), proposed a method time synchronization technique for the detection and handling of Black hole attack in wireless sensor network. We have also calculated its result using performance metrics like throughput, packet loss and efficiency. On the behalf of results of trace graphs, we can conclude that in case of time synchronization technique throughput of received packet is quite high when there is black hole attack in the network.

By Lalwani et al. (2018) depicts implementation details of prototype scheme of threat detection in wireless sensor systems. The framework design and innovations used to accomplish given feature set were introduced. Utilizing mobile platform for security components from the software utilized by nodes in the secured system which is positive feature when managing sensor's battery life. The secluded design and uniform organization of investigated messages is a beginning stage for making new techniques for threat identification. Now another research in behavioural investigation of WSN nodes is arranged so as to make a state machine compliant to the WSN protocol stack.

By Ibarra et al. (2016), depicts that a novel security models to resolve three significant active attack termed as MITM attack, cloning attack and replay attack. The idea of zero knowledge protocol is utilized which guarantees non-transmission of crucial data amongst verifier and prover. The anticipated model utilizes social finger print based on s-disjunct code together with ZKP to identify clone attacks and eliminate replay and MITM attack. This is also extended the scheme using work rate to evaluate 
the clone node detection. Various attack scenarios also analyzed, i.e., performance and cryptographic strength of proposed model.

In this study, the existing clustering based routing schemes have been discussed. Furthermore, the plans benefits and faults are portrayed. The previously mentioned calculations depend on single-hop clusters in which the cluster individuals are one-hop far from the $\mathrm{CH}$. Vehicles just join the clusters where the $\mathrm{CH}$ is in its neighbourhood regions. One-hop cluster topology can diminish the cluster re-association and abatement cluster support overhead because fewer information exchanges are requisite. Though, the transmission range and thickness of vehicles influence the measure of cluster. In a high vehicular thickness, information collision may occur in the clusters. Unexpectedly, a vehicle may neglect to recognize neighbours in low thickness. Newly, a lot of works have been proposed for multi-hop clustering calculations.

Mekikis et al. (2014) was planned to outline inert clustering aided routing protocol for vehicular ad hoc networks (PassCAR). It was gone for routing and choosing the following hub for transmitting data in view of Route Request (RREQ) messages. In PassCAR, every hub makes sense of its need as per the measurements, for example, hub degree, expected transmission count and link lifetime. In accordance with these needs, this technique produces steady and strong clusters amid the route discovery process in order to enhance network throughput. Though, this protocol was less appropriate for urban situation which was credited to its broadcasting strategy for finding routes which can result in notable routing overhead.

Hashmi et al. (2010) anticipated prior forwarding strategy describes first routing decision of protocol when there are packets to be sent. In Delay Bounded protocols prior forwarding strategy is utilized, in all other routing protocols wireless multi hop technique for sending is utilized. Digital map offers traffic statistics and street level map like vehicle speed and traffic density on road at different times. Digital map is essential in case of certain cluster based routing protocols (Purohit et al., 2010). Virtual Infrastructure is made through nodes cluster so as to give scalability. Each group can have cluster head which is responsible for secure communication between inter-cluster and intra cluster coordination in network. Recovery technique is utilized to recover from unfavourable situations. Recovery procedure is criteria which is utilized to judge the execution of protocol.

Hezaveh et al. (2015) proposed a proficient prediction-based data forwarding technique to give efficient and reliable data transmission in vehicular ad hoc networks. A new link utility expectation algorithm is given by utilizing vehicle speed and information about location. Zhu et al. demonstrated that when the proposed forwarding strategy was brought into routing protocol, better packet delivery ratio and end-to-end delay were accomplished. Moreover, proposed link utility variation with two-hop neighbour data to enhance reliability of selected transmission path. At long, effects of some key parameters are investigated. Iterative results demonstrated that routing protocols with forwarding schemes accomplish reduced end-to-end delay, particularly in high density. Average amount of hops between source and destination pair was diminished without expanding packet loss probability about by link breakage. The effectiveness of data transmission was enhanced as demonstrated by diminished end to end delay.

By Shankar and Shanmugavel (2014) present root kit and malware discovery framework to secure hidden virtualization infrastructure against digital assaults in attacks in cloud security approaches, it utilizes framework associated with hashing and also the utilization of SVM together with VMI. The utilization of a secured in-VM screen guarantees that the inner visitor VM state can be precisely acquired without it being imperilled while the utilization of a disconnected SVM classifier in remote control monitoring takes into consideration rapid attack classification.

By Sherubha and Priya (2016) focuses on the sensor technology used to generate condition monitoring data to enable practical situation monitoring schemes. This information must be deal with and turned into practical data to produce practical information. Condition monitoring schemes must store huge quantities of information to construct models for examination. The data must be validated first to promise that they are precise and error-free (sensor errors, noise, null values, communication faults, etc.). This technique may even be carried out in the sensor node's microcontroller consequently, merely legitimate data are broadcasted hence reducing the transmission load. This information can then be practiced in a numeral of diverse ways to produce information. Once, information is collected, they can be examined by means of robust algorithms to recognize faults in near real time. Algorithms necessitate being robust as WSN information is noisy can be irregular, may enclose errors has numerous interdependencies and the information volume is extremely high. The WSN information can also be accumulated and examined over longer time periods to recognize long term progressive faults like slowly rising crack. 
By Narmatha et al. presented a comprehensive review of WSN based SHM systems. Background information relating to structural health monitoring such as common sensors, commonly measured parameters and damage detection and localization algorithms were discussed. The main challenges of scalability, time synchronization, sensor placement optimization and data processing were presented and solutions to these problems discussed and compared. Experimental work performed in the lab and on real-world structures was presented and discussed. Finally, future research directions for SHM systems using WSNs were presented.

\section{MATERIALS AND METHODS}

Network model: Consider a sensor network, in which ' $N$ ' sensing nodes are independently deployed and uniformly distributed in dimensional region $\mathrm{R}(\mathrm{x}, \mathrm{y})$ and base station is located outside the sensor region (Sherubha and Priya, 2016). In general, there is no prior knowledge regarding the position of $\mathrm{BS}$, hence, distributing the nodes randomly and deploying is an effectual strategy. In this investigation, random distribution is provided based on Eq. 1:

$$
P(N(R)=K)=\frac{\left(\lambda|R|^{k} \exp -\lambda|R|\right)}{K !}
$$

Where:

$\lambda=$ The node density

$|R|=$ The region of sensor network

In this network modelling, a node accumulates information from surrounding environment and propagates this information to base station with the assistance of cluster head (Sherubha and Priya, 2016). Therefore, in this method, there are three kinds of nodes in sensing nodes, cluster head nodes and base station.

Consider ' $\mathrm{N}$ ' is set of field nodes, cluster head is set of cluster head nodes. Here, $N=\left\{j \mid j \geq 1 \mathrm{Vj} \leq \mathrm{j}_{\max }\right\}$ and $\mathrm{j}_{\max }$ is maximum amount of nodes. Cluster heads $=\{\mathrm{CH} \mid \mathrm{CH} \geq 1 \mathrm{~V}$ $\mathrm{CH} \leq \mathrm{CH} \_$max $\}$where $\mathrm{Ch}_{\max }$ is maximum amount of cluster head. Every field node is capable to set a link to other field nodes or cluster head based on the situation. With the above discussion, connectivity parameter is found in Eq. 2:

$$
P_{a, b}= \begin{cases}1 & \text { If ' } a \text { ' establishes connection with ' } b \text { ' } \\ 0 & \text { Otherwise }\end{cases}
$$

If $\mathrm{a}, \mathrm{b} \in \mathrm{N}$ and $\mathrm{a} \neq \mathrm{b}$. Here, $\mathrm{b}$ may be either cluster head or member node. If ' $\mathrm{b}$ ' is a cluster head, then ' $\mathrm{a}$ ' is member node. Link quality is directly associated with distance and received signal strength (Manikandan et al., 2018).

Cuckoo search for establishing route: In this study, initially fitness function has to estimate route for data transmission using cluster head selection and to describe cost function for evaluating cuckoo nest is discussed. Subsequently, the anticipated Cuckoo search based Routing Establishment (CS-REA) is presented.

Fitness function derivation: For selecting an appropriate cluster head, fitness function of every node is evaluated with Eq. 3:

$$
\text { fitness }=\mathrm{a}_{1} * \mathrm{ff}_{1}+\mathrm{a}_{2} * \mathrm{ff}_{2}+\mathrm{a}_{3} * \mathrm{ff}_{3}
$$

where, $\mathrm{a}_{1}, \mathrm{a}_{2}$ and $\mathrm{a}_{3}$ constant and its value are lies between 0 and $1 . a_{3}$ value equal to $1-a_{1}-a_{2}$ as in Eq. 4 :

$$
\mathrm{ff}_{1}=\frac{1}{\mathrm{~m}} *\left[\frac{\sum_{\mathrm{i}=1}^{\left|\mathrm{c}_{\mathrm{n}}\right|} \operatorname{distan} \operatorname{ce} \beta\left(\mathrm{CH}_{\mathrm{w}} \mathrm{CM}_{\mathrm{i}}\right)}{\left|\mathrm{c}_{\mathrm{n}}\right|}\right]
$$

Here, ' $m$ ' is the amount of cluster heads, $c_{n}$ is amount of cluster members in nth cluster and distance $\left(\mathrm{CH}_{\mathrm{n}}, \mathrm{CM}_{\mathrm{i}}\right)$ is the distance nth cluster head and ith cluster member. Subsequently, $\mathrm{ff}_{2}$ of fitness function is specified in Eq. 5:

$$
\mathrm{ff}_{2}=\frac{E_{\text {residual }}}{E_{\text {total }}}
$$

Where:

$$
\begin{aligned}
\mathrm{E}_{\text {residual }}= & \text { The nodes residual energy } \\
\mathrm{E}_{\text {total }}= & \text { The is total nodes energy that lies in } \\
& \text { communication range of particles }
\end{aligned}
$$

Next, $\mathrm{ff}_{3}$ is the fitness function that is expressed in Eq. 6 :

$$
\mathrm{ff}_{3}=\frac{\mathrm{c}_{\mathrm{n}}}{\mathrm{N}}
$$

Where:

$c_{n}=$ The No. of average cluster member in a specific cluster

' $\mathrm{N}$ ' = The total amount of nodes

After evaluating the fitness function of every node, almost $20 \%$ node is chosen that possess highest fitness value. From the available $20 \%$ nodes, eligible nodes are chosen as cluster head (Sivaram et al., 2018a-c) thereby, 
every cuckoo nest is populated. It means, eligible nodes are candidate cluster heads that functions as cuckoo's egg which is utilized for host nest initialization. For choosing an optimal nest, cost of every host nest is examined based on below given cost function as in Eq. 7 :

$$
\text { Function }_{\text {cost }}=\gamma^{*} \mathrm{x}_{1}+(1+\gamma)^{*} \mathrm{x}_{2}
$$

The cost function sub sets like $\left(\mathrm{x}_{1}, \mathrm{x}_{2}\right)$ is provided as in Eq. 8 and 9:

$$
\begin{gathered}
\mathrm{x}_{1}=\max _{\mathrm{i}=1,2,3, \ldots, \mathrm{I}}\left\{\sum \mathrm{s}\left(\mathrm{n}_{\mathrm{i}}, \mathrm{CH}_{\mathrm{e}, 1}\right) /\left|\mathrm{C}_{\mathrm{e}, 1}\right|\right\} \\
\mathrm{x}_{2}=\frac{\sum_{\mathrm{i}=1}^{\mathrm{N}} \mathrm{E}\left(\mathrm{n}_{\mathrm{i}}\right)}{\sum_{\mathrm{i}=1}^{\mathrm{i}} \mathrm{E}\left(\mathrm{CH}_{\mathrm{e}, \mathrm{i}}\right)}
\end{gathered}
$$

Here, function $\mathrm{x}_{1}$ specifies maximum average Euclidean distance amongst sensor nodes $\left(\mathrm{n}_{\mathrm{i}}\right)$ and the related cluster head. $\mathrm{CH}_{e, 1}$ specifies sum of nodes that is available in communication range of cluster head $\mathrm{c}_{\mathrm{i}}$ of egg ' $\mathrm{e}$ '. $\mathrm{x}_{2}$ determines the function of ratio of total amount of nodes to total amount of energy consumed by the nodes connected in the network. Thus, total energy consumed by cluster head present in the cuckoo nest ' $e$ '. The $\gamma$ value is approximately 0.5 . In which $\mathrm{x}_{1}$ and $\mathrm{x}_{2}$ are minimum intra cluster distance and to choose optimal location based on cluster head which reduces the energy consumed by sensor nodes (Algorithm 1).

\author{
Algorithm 1; Sensor node: \\ 1. Begin \\ 2. Construct initial population of $\mathrm{n}$ nests (solutions) \\ 3. Define objective function $\mathrm{f}(\mathrm{x})$ \\ 4. Set range max, min \\ 5. Set range of the nest: Xmin, Xmax \\ 6. Set maximum number of iterations: $\mathrm{N} \_$itertotal \\ 7. For all $\mathrm{x}_{\mathrm{i}}$ do \\ 8. Evaluate fitness $F_{i}=f\left(x_{i}\right)$ \\ 9. End For \\ 10. N_iter $=1$ \\ 11. While $(\mathrm{N}$ iter $<\mathrm{N}$ itertotal) do \\ 12. For all $\mathrm{x}_{\mathrm{i}} \mathrm{do}$ \\ 13. Evaluate step size for Levy flight using Eq. 4 \\ 14. Generate a new cuckoo from nest randomly by Levy flight \\ 15. If $\left(x_{j}>X_{\max }\right)$ then \\ 16. $\mathrm{X}_{\mathrm{j}}-\mathrm{X}_{\max }$ \\ 17. End If \\ 18. If $\left(\mathrm{x}_{\mathrm{i}}<\mathrm{X}_{\text {min }}\right)$ then \\ 19. $\mathrm{x}_{\mathrm{j}}-\mathrm{X}_{\min }$ \\ 20. End If \\ 21. Compute fitness \\ 22. Choose a random nest among $n$ nest randomly \\ 23. If $\left(\mathrm{F}_{\mathrm{j}}>\mathrm{F}_{\mathrm{k}}\right)$ then \\ 24. $\mathrm{x}_{\mathrm{j}}+\mathrm{x}_{\mathrm{k}}$ \\ 25. $\mathrm{F}_{\mathrm{j}}-\mathrm{F}_{\mathrm{k}}$ \\ 26. End If \\ 27. End For \\ 28. Maintain global optimal fitness
}

29. Compute probability using Eq. 7

30. Fraction of worse nests and new ones/solutions are built

31. If $\left(\mathrm{x}_{\mathrm{P}}>\mathrm{X}_{\max }\right)$ then

32. $\mathrm{X}_{\mathrm{i}}-\mathrm{X}_{\max }$

33. End If

34. If $\left(x_{i}<X_{\min }\right)$ then

35. $\mathrm{X}_{\mathrm{i}}-\mathrm{X}_{\min }$

36. End If

37. Evaluate quality/fitness

38. Keep best solutions

39. End For

40. Rank all the solutions

41. Construct markv model

42. find current best node

43. Evaluate next successive transition

44. Perform multi-hop in distributed environment

45. Successful transmission

46. End for

47. End if

Markov Model: In this study, Markov Model is used to design the state of sensor nodes in randomly distributed sensor environment. This model investigates the non-linear, highly dynamic systems in accordance to the discrete events of node state. In this wireless sensor communication scenario, mobility rate of nodes and energy consumption rate of node can be estimated jointly using Received Signal Strength metric at sink node. This estimation is utilized in formulating probability density matrix. Here, Markov chain models the nodes energy state based on the location of nodes position. This location may change due to communication epoch (Sivaram et al., $2018 \mathrm{a}-\mathrm{c}$ ). This state can be designed as stochastic nature, comprising set of possible event occurrence of nodes to transmit data to destination in which probability of every node is described by state of information attained in previous event. This state computation of nodes assists in determining the route for next possible hop and energy consumed by nodes with state transition representing next hop (Fig. 2).

For certain sensor network deployment, let ' $N$ ' node position or energy states. Then, the set of states for every node, $N=\left\{n_{1}, n_{2}, \ldots, n_{m}\right\}$.

Combined state transition of sensor node position and nodes subsequent states are illustrated using the Markov chain as given in Fig. 3. After that, hidden Markov model is generally defined with following notations.

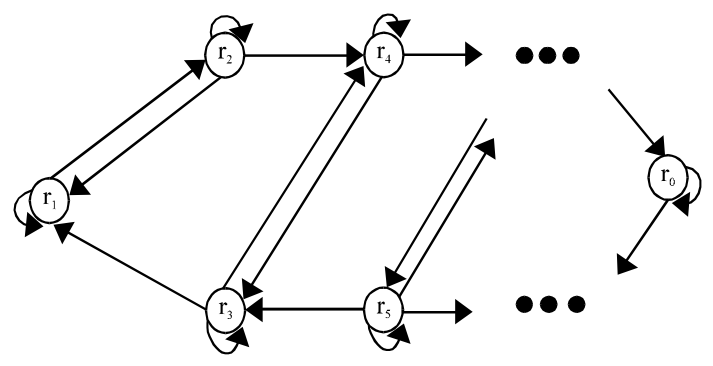

Fig. 2: State transition diagram 


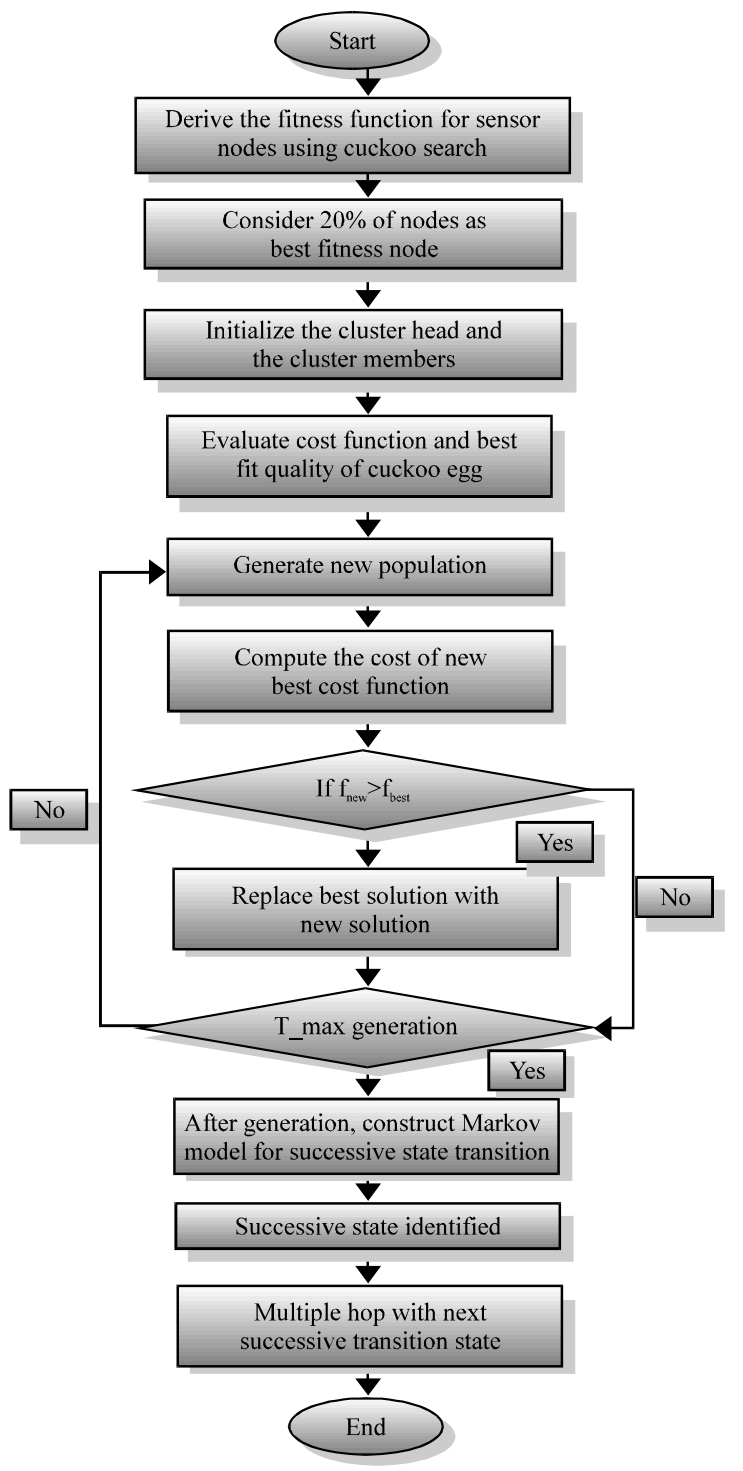

Fig. 3: Flow diagram of proposed CMMRR techniquef

Where, $\mathrm{N}$ specifies Markov chain's state space and defined as set ' $N$ ' comprising diverse node positions for next transition and energy consumed by nodes to specify fractional changes in Received Signal Strength metric. ' $M$ ' is transition matrix in association with present state of nodes for next transition and energy consumed by probability of same route (Porkodi et al., 2018) through specific state vector in row ' $M$ ' and ' $\pi$ ' is member of ' $R$ " specifying vector of initial state transition. Transition probability and probability matrix ' $\pi$ ' are designed using samples of collected data. Matrix ' $M$ ' can be identified as in Eq. 10:

$$
\mathrm{M}\left[\mathrm{N}_{\mathrm{i}}, \mathrm{N}_{\mathrm{j}}\right]=\frac{\mathrm{A}\left(\mathrm{r}_{\mathrm{i}}, \mathrm{r}_{\mathrm{j}}\right)}{\sum \mathrm{A}\left(\mathrm{r}_{\mathrm{i}}, \mathrm{r}_{\mathrm{j}}\right)}
$$

where, $A\left(r_{i}, r_{j}\right)$ specifies how the subsequent routes are followed by $r_{j}$ by the node $r_{i}$. Moreover, $\pi[r]$ can be distinct as shown in Eq. 11:

$$
\pi[\mathrm{r}]=\frac{\mathrm{A}\left(\mathrm{r}_{\mathrm{i}}\right)}{\sum \mathrm{A}\left(\mathrm{r}_{\mathrm{k}}\right)}
$$

where, $A\left(r_{i}\right)$ determines how the next transition $r_{i}$ was encountered. The next transition state for routing is selected with state probability vector to predict next state of vector distribution. Subsequently, $\pi$ is vector state probability as in Eq. 12:

$$
\pi^{\mathrm{n}}=\pi^{*} \mathrm{M}^{\mathrm{n}}
$$

where, $\mathrm{M}^{\mathrm{n}}$ specifies next $\mathrm{n}$-step transition routing state. This computation results further in next state vector probability $\pi^{\mathrm{n}}$ as provided in Eq. 13:

$$
\mathrm{R}^{\mathrm{n}}=\operatorname{Maximum}\left[\mathrm{r}_{1}^{\mathrm{n}}, \mathrm{r}_{2}^{\mathrm{n}}, \mathrm{r}_{3}^{\mathrm{n}}, \ldots, \mathrm{r}_{\mathrm{k}}^{\mathrm{n}}\right]
$$

Along with this, differential gain or change in Received Signal Strength (RSS), $\beta$ which is utilized to verify whether nodes transition from one node to another node. The positive value of differential gain is node moving to next state of cluster head. As both differential gain and transition matrix are related with route determination, these factors are utilized to predict future route for sensor node. If differential gain ' $\beta$ is zero, in successive route measurement, then node has not moved in route with time.

The above combined transition matrix ' $\mathrm{N}$ ' in discrete time sequence ' $t$ ' is $\{\mathrm{N}) \mathrm{t}$ and differential gain measurement $\beta$ provides precise node location as in Eq. 14:

$$
\text { Probability }=\left[\mathrm{V}^{\mathrm{T}} \cdot \mathrm{N}^{\mathrm{t}}\right] \cdot{ }^{*} \gamma, \mathrm{t}=1,2, \ldots, \infty
$$

Where:

$\mathrm{v}=$ The vector representing the current state of the nodes position

$\delta=$ The vector of differential RSS gain for all sensor nodes in the network

The operation $\left(.^{*}\right)$ determines the precise location of each node based on which packet transmission is accomplished (Sherubha and Priya, 2016). The vector $\delta$ in Eq. 9 is differential gain in RSS, for all nodes.

Where, ' $v$ ' specifies vector determining current state of node location and $\beta$ specifies differential vector of RSS gain for entire sensor nodes in the network. Operations like $\left({ }^{*}\right)$ specifies accurate route of every node based on packet transmission is achieved. Vector $\beta$ in Eq. 15 and 16 is RSS based differential gain for entire nodes: 


$$
\begin{gathered}
\gamma_{\mathrm{i}}=\mathrm{f}\left(\mathrm{i}, \gamma_{\mathrm{t}}\right) \quad \mathrm{f}\left(\mathrm{i}, \gamma_{\mathrm{t}}\right)>0 \\
\gamma_{\mathrm{i}}=0 \quad \mathrm{f}\left(\mathrm{i}, \gamma_{\mathrm{t}}\right)<0
\end{gathered}
$$

where, $\mathrm{f}\left(\mathrm{i}, \gamma_{\mathrm{t}}\right)<$ is RSSI difference value at discrete successive time. Eq. 17 is defined as:

$$
f\left(i, \gamma_{t}\right)=\left[\operatorname{RSSI}\left(i, t_{k+1}\right)-\operatorname{RSSI}\left(i, t_{k}\right)\right]
$$

\section{RESULTS AND DISCUSSION}

In the study, the simulation setup and the numerical results are discussed in detail. The simulation was carried out in MATLAB environment. The parameters associated with the simulation of cuckoo based Markov model for routing algorithm is given in Table 1 . The ultimate goal of this investigation is to predict the routing mechanism by predicting the successive transition state of nodes and to reduce the packet drop while executing the anticipated method. Performance metrics of this research is given in terms of end to end delay, success delivery rate and power consumption for various sized network. The mobility pattern of nodes is configured as random distribution by placing 25 nodes in a network dimension of $100^{*} 100$

The simulation outcome of the cuckoo based Markov model for reliable routing algorithm without and with Markov chain for nodes energy state and localization scenario is compared with optimal clustering concept. The comparison of the proposed CMMRR is done with the Efficient and Reliable routing algorithm (EAR) in accordance with the performance metrics like success delivery rate, end to end delay and power utilization as in Table 2 and 3 .

This research depicts least delay which is compared with two other protocols and can be seen in below given Fig. 4. This is done with the organization of sensor networks in adaptive clusters which considerably eliminated traffic congestion for data traffic.

From Fig. 5 it is understood that the success delivery rate of the proposed CMMRR model is higher when compared with the prevailing EAR algorithm devoid of any statistical Markov chain model. In the prevailing EAR, even though energy abundant nodes with shortest distance scenario is considered while setting efficient and reliable RF links as in Table 4, precise node positioning factors are not addressed as in Fig. 6. Meanwhile, in the execution assume a scenario where nodes actual location identification is carried out periodically to form robust and

\begin{tabular}{|c|c|c|c|}
\hline \multicolumn{2}{|l|}{ Parameters } & \multicolumn{2}{|l|}{ Specifications } \\
\hline \multicolumn{2}{|l|}{ Network area } & \multicolumn{2}{|l|}{$100 * 100$} \\
\hline \multicolumn{2}{|l|}{ Sensor nodes } & \multicolumn{2}{|l|}{$5,10,15,20,25$} \\
\hline \multicolumn{2}{|l|}{ Clusters } & \multicolumn{2}{|l|}{4} \\
\hline \multicolumn{2}{|c|}{ Transition state } & \multicolumn{2}{|c|}{4 (sleep, idle, active, supervisor) } \\
\hline \multicolumn{2}{|c|}{ Mobility model } & \multicolumn{2}{|c|}{ Random distribution } \\
\hline \multicolumn{2}{|c|}{ Protocol } & \multicolumn{2}{|l|}{ IEEE 802.11} \\
\hline \multicolumn{2}{|l|}{ Traffic } & \multicolumn{2}{|l|}{$10 \mathrm{~B} /$ packet } \\
\hline \multicolumn{2}{|l|}{ Packet size } & \multicolumn{2}{|l|}{$10 \mathrm{~KB}$} \\
\hline \multicolumn{2}{|l|}{ Interface type } & \multicolumn{2}{|l|}{ Wireless } \\
\hline \multicolumn{2}{|l|}{ Frequency } & \multicolumn{2}{|l|}{$2.4 \mathrm{GHz}$} \\
\hline \multicolumn{4}{|c|}{ Table 2: E2E computation } \\
\hline & \multicolumn{3}{|c|}{ End to End delay computation } \\
\hline No. of nodes & Without Markov Model & With Markov Model & EAR \\
\hline 5 & 0.9 & 0.3 & 0.5 \\
\hline 10 & 1.3 & 0.7 & 1.2 \\
\hline 15 & 1.5 & 1.1 & 1.2 \\
\hline 20 & 2.2 & 1.7 & 1.8 \\
\hline$\underline{25}$ & 3.4 & 2.2 & 2.3 \\
\hline
\end{tabular}
reliable RF links towards destination.
Table 1: Simulation parameter and specifications

Table 3: Success delivery rate computation

\begin{tabular}{lccc}
\hline \multicolumn{4}{c}{ Success (Packet) delivery rate } \\
No. of nodes & Without Markov model & With Markov model & EAR \\
\hline 5 & 85 & 94 & 93 \\
10 & 83 & 91 & 89 \\
15 & 81 & 90 & 85 \\
20 & 79 & 85 & 81 \\
25 & 73 & 78 & 77 \\
\hline
\end{tabular}

Table 4: Success delivery rate computation Power utilization while transition

\begin{tabular}{lccc} 
No. of nodes & Without Markov model & With Markov Model & EAR \\
\hline 5 & 11 & 5.5 & 08 \\
10 & 17 & 12.0 & 13 \\
15 & 28 & 19.0 & 21 \\
20 & 30 & 23.0 & 25 \\
25 & 31 & 29.0 & 31 \\
\hline
\end{tabular}

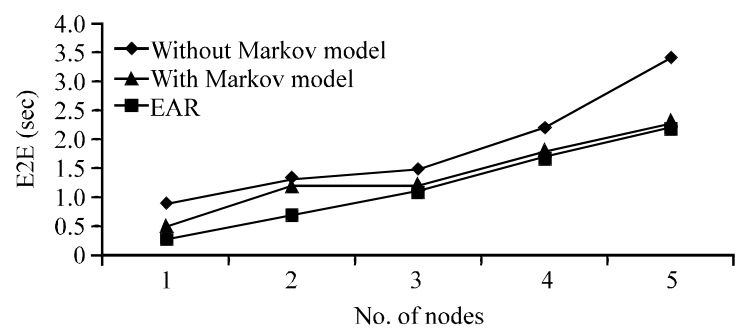

Fig. 4: Graphical representation of E2E computation

At last, power utilized by every transition nodes in the Markov Chain model in the anticipated CMMRR approach shows an enhancement with a range of $10.4-29 \%$ over the prevailing EAR protocol. With respect to improved throughput in anticipated technique, it shows an outcome with minimal power expanded for retransmission as in Fig. 7 and 8. As well, network energy is preserved owing to load balancing amongst the nodes which is carried out in optimal and dynamic manner. 


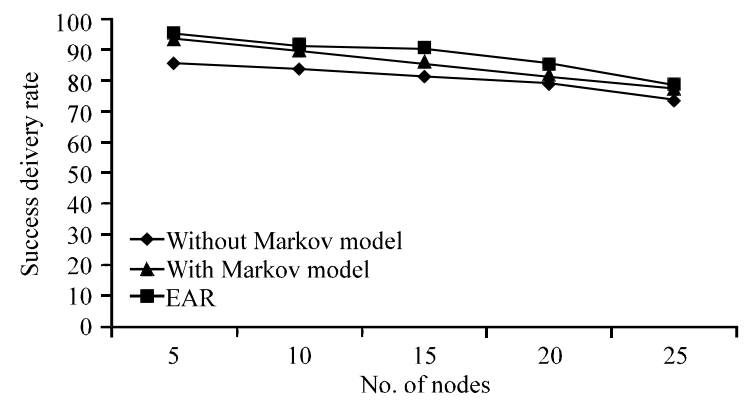

Fig. 5: Graphical representation of SDR computation

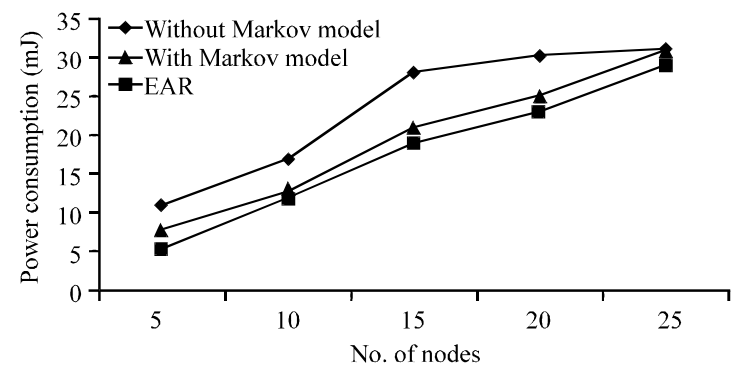

Fig. 6: Graphical representation of power computation

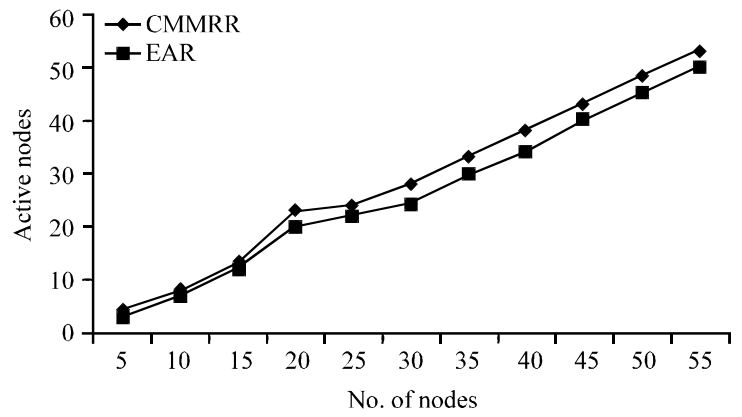

Fig. 7: Graphical representation of Alive nodes in network

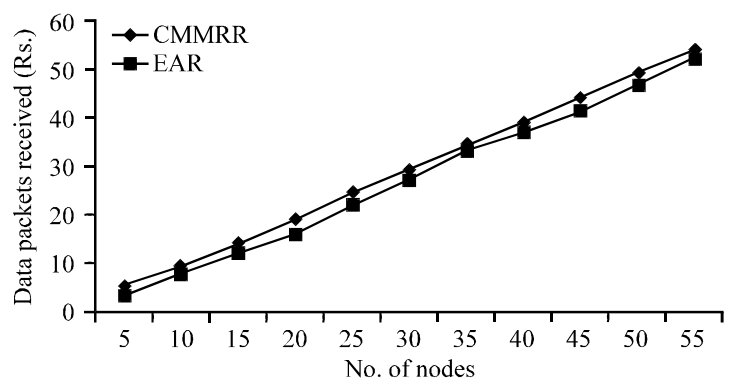

Fig. 8: Graphical representation of data transmission in network

\section{CONCLUSION}

In this investigation, an effectual Cuckoo for Markov Model based Reliable Routing (CMMRR) model is anticipated for balancing energy amongst the successive nodes and to identify the next optimal state of promoting routing, thereby fitness function is derived using cuckoo search model. The proposed algorithm shows a random distribution with the cluster head uniformly in all the directions, so that, communication load is distributed randomly. So, as to transmit the aggregated data from cluster head to sink node, multi hop transition of successive node has to be estimated for choosing an energy effectual schemes like EAR are discussed.

As, energy be a scarcest resources in WSN during routing process, a significant part of it is exhausted during the idle state of certain node while data communication between the nodes. Henceforth, it is crucial to reduce redundant retransmission and packet drop information in the randomly distributed WSNs. This can be attained with suitable and appropriate amount of nodes for routing the packets without burdening the successive state of routing nodes while propagation. This can be achieved by observing the energy model of nodes and positioning of every node periodically and generating probability transition matrix. The discrete Markov chain model is utilized along with cuckoo search to identify the successive states of sensor nodes by evaluating the current node. The utilization of this stochastic model eliminated exchange of control signals for power scheduling, localization in dynamic network scenario. Simulation outcomes demonstrate that the anticipated method provides minimum power consumption devoid of affecting nodes life time and end to end delay. This also enhances the network life time of nodes and effectually balances sensor node's energy level in network.

Simulation outcomes depicts that the anticipated CMMRR strategy perform well than the conventional EAR strategy with respect to total energy consumption, energy, routing efficiency and nodes life time. This research can also be further extended in multi-3D model of WSN and the performance metrics can be observed during the presence on network mobility.

\section{REFERENCES}

Abuarqoub, A., M. Hammoudeh, B. Adebisi, S. Jabbar and A. Bounceur et al., 2017. Dynamic clustering and management of mobile wireless sensor networks. Comput. Networks, 117: 62-75.

Ghaffari, A. and S. Nobahary, 2015. FDMG: Fault detection method by using genetic algorithm in clustered wireless sensor networks. J. AI Data Min., 3: 47-57.

Ghaffari, A., 2014. Designing a wireless sensor network for ocean status notification system. Indian J. Sci. Technol., 7: 809-814. 
Hashmi, S.U., S.M.M. Rahman, H.T. Mouftah and N.D. Georganas, 2010. Reliability model for extending cluster lifetime using backup cluster heads in cluster-based wireless sensor networks. Proceedings of the 2010 IEEE 6th International Conference on Wireless and Mobile Computing, Networking and Communications, October 11-13, 2010, IEEE, Niagara Falls, Canada, ISBN:978-1-4244-7743-2, pp: 479-485.

Hezaveh, M., Z. Shirmohammdi, N. Rohbani and S.G. Miremadi, 2015. A fault-tolerant and energy-aware mechanism for cluster-based routing algorithm of WSNs. Proceedings of the 2015 IFIP/IEEE International Symposium on Integrated Network Management (IM), May 11-15, 2015, IEEE, Ottawa, Canada, ISBN:978-1-4799-8241-7, pp: 659-664.

Ibarra, E., A. Antonopoulos, E. Kartsakli, J.J. Rodrigues and C. Verikoukis, 2016. QoS-aware energy management in body sensor nodes powered by human energy harvesting. IEEE. Sens. J., 16: 542-549.

Kuila, P. and P.K. Jana, 2014. A novel differential evolution based clustering algorithm for wireless sensor networks. Appl. Soft Comput., 25: 414-425.

Lalwani, P., H. Banka and C. Kumar, 2018. BERA: A biogeography-based energy saving routing architecture for wireless sensor networks. Soft Comput., 22: 1651-1667.

Mahalakshmi, K., R. Arun, P. Sherubha and K. Sindhanaiselvan, 2017. Dynamic virtual machines building utilizing KBR agent for information security in hybrid cloud. J. Adv. Res. Dyn. Control Systems, 1: 1181-1190.

Manikandan, V., V. Porkodi, A.S. Mohammed and M. Sivaram, 2018. Provacy preservation data mining using threshold based fuzzy C- means clustering. ICTACT. J. Soft Comput., 9: 1820-1823.

Mann, P.S. and S. Singh, 2017. Energy efficient clustering protocol based on improved metaheuristic in wireless sensor networks. J. Network Comput. Appl., 83: 40-52.

Mekikis, P.V., A.S. Lalos, A. Antonopoulos, L. Alonso and C. Verikoukis, 2014. Wireless energy harvesting in two-way network coded cooperative communications: A stochastic approach for large scale networks. IEEE. Commun. Lett., 18: 1011-1014.
Mottaghinia, Z. and A. Ghaffari, 2016. A unicast tree-based data gathering protocol for delay tolerant mobile sensor networks. J. Inf. Syst. Telecommun., 59: 1-12.

Porkodi, V., M. Sivaram, A.S. Mohammed and V. Manikandan, 2018. Survey on white box attacks and solutions. Asian J. Comput. Sci. Technol., 7: 28-32.

Purohit, N., A. Verma and H. Agrawal, 2010. Performance analysis of wireless sensor network with virtual MIMO. Proceedings of the 2010 Annual IEEE India Conference on (INDICON), December 17-19, 2010, IEEE, Kolkata, India, ISBN:978-1-4244-9072-1, pp: 1-4.

Shankar, T. and S. Shanmugavel, 2014. Energy optimization in cluster based wireless sensor networks. J. Eng. Sci. Technol., 9: 246-260.

Sherubha, P. and M.M. Priya, 2016. A detailed survey on security attacks in WSN. Intl. J. Soft Comput., 2016: 221-226.

Sherubha, P., 2018. Energy efficient and bandwidth aggregation techniques in wireless heterogeneous devices: A survey. Intl. J. Pure Appl. Math., 118: 291-300.

Shokouhifar, M. and A. Jalali, 2017. Optimized sugeno fuzzy clustering algorithm for wireless sensor networks. Eng. Appl. Artif. Intell., 60: 16-25.

Sivaram, M., A.S. Mohammed, V. Porkodi and V. Manikandan, 2018b. Securing the sensor networks along with secured routing protocols for data transfer in wireless sensor networks. J. Emerging Technol. Innovative Res., 5: 316-321.

Sivaram, M., D. Yuvaraj, A.S. Mohammed, V. Porkodi and V. Manikandan, 2018a. The real problem through a selection making an algorithm that minimizes the computational complexity. Intl. J. Eng. Adv. Technol., 8: $95-100$.

Sivaram, M., D. Yuvaraj, V. Porkodi and V. Manikandan, 2018. Emergent news event detection from Facebook using clustering. J. Adv. Res. Dyn. Control Syst., 1: $1941-1947$. 